Memoirs of the College of Science, University of Kyoto, Series A

Vol. XXX, Mathematics No. 2, 1957.

\title{
Le probleme de Cauchy pour les équations hyperboliques
}

Par

Sigeru Mizohata

(Reçu le 2 Août 1956)

1. Introduction. Dans son cours intitulé 《hyperbolic differential equations》 ([1]), M. Leray a développé une théorie des équations hyperboliques en se fondant sur la transformation de Laplace. Ce petit article à pour but de montrer que, tant qu'on considère l'équation hyperbolique donnée comme une équation d'évolution (Voir [2]), on peut faire un raisonnement assez simple pour le problème de Cauchy. En effet, M. Leray a trouvé une matrice, et grace à cette matrice, il a étendu l'inégalité d'énergie de Friedrichs et Lewy pour des solutions assez régulières. Nous allons montrer que l'inégalité d'énergie subsiste même dans le cas où des solutions sont distributions mais non pas fonctions. Nous discuterons dans un autre article le problème de Cauchy pour les équations paraboliques en nous appuyant sur presque le même principe.

2. Opérateur. $\tau_{11}(p) \quad(-\infty<q<+\infty)$. Soit $f$ tempérée, alors on définit $\tau_{\eta}(p) f \equiv\left(1-p_{1}{ }^{\prime}-\cdots-p_{n}{ }^{\prime \prime}\right)^{\eta} f$ comme l'image réciproque de Fourier de $\left(1+4 \pi^{2}|\xi|^{2}\right)^{q} \varphi_{\xi} \epsilon\left(\phi^{\prime}\right)_{\xi}, \varphi_{\xi}$ étant la transformée de $f$. (Voir [1], [3]).

Espace hilbertien $D^{s}(-\infty<s<+\infty) . \quad f \in D^{s}$, si $f$ est tempérée et $\tau_{* / 2}(p) f \in L^{*}$. On le munit du produit scalaire suivant:

$$
(f, g)=\left(\tau_{s / 2}(p) f, \tau_{* / 2}(p) g\right)=\left(\tau_{s}(p) f, g\right) .
$$

Remarquons que, si $s^{\prime}<s$, alors $D^{{ }^{\prime}} \supset D^{s}$, c'est-à-dire que $D^{s}$ est contenu dans $D^{s^{\prime}}$ avec une topologie plus fine.

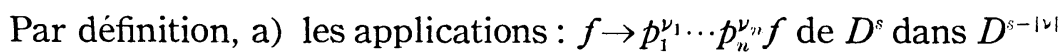
b) les applications: $f \rightarrow \tau_{\eta}(p) f$ de $D^{s}$ dans $D^{\varsigma-2}$, sont des applications continues.

Nous aurons besoin ultérieurement le 
LEMME 1. Soit q entier positif quelconque, $f \in\left(\bigotimes_{L^{2}}^{\prime}\right)$ et $c(x) \in(\mathcal{B})$. Alors

$(2 \cdot 1) \tau_{-q}(p) c(x) f=c(x) \tau_{-q}(p) f+\sum_{\substack{|| 2 \mid \leq k, k \\ k=1, a, \cdots, q}} p_{1}^{\nu_{1}} \cdots p_{n}^{\nu_{n} \tau_{-k}}(p) c_{\nu, k}^{\prime}(x) \tau_{-q}(p) f$, out $c_{v, k}^{\prime}(x)$ sont des combinaisons linéaires des dérivées de $c(x)$.

LEMME 2. Soit s entier quelconque. $a(x) \in(\mathcal{B}), f \in D^{s}$. Alors, l'application $(a(x), f) \rightarrow a(x) f d e(\mathcal{B}) \times D^{*}$ dans $D^{s}$ dans $D^{s}$ est continue.

Démonstration du lemme 1. $\quad\left(1-p_{1}{ }^{1}-\cdots p_{n}{ }^{2}\right) c(x) f=c(x)\left(1-p_{1}{ }^{2}-\right.$ $\left.\cdots p_{n}{ }^{2}\right) f-\left[\sum_{i=1}^{n} p_{i}{ }^{2} c(x)\right] f-2 \sum_{i=1}^{n}\left[p_{i} c(x)\right] p_{i} f$. En remplaçant $f$ par $(1-$ $\left.p_{1}{ }^{2}-\cdots-p_{n}{ }^{2}\right)^{-1} f$ et en appliquant $\tau_{-1}(p)$ à gauche,

$$
\begin{gathered}
c(x) \tau_{-1}(p) f=\tau_{-1}(p)\left[c(x)-\left\{\sum_{i=1}^{n} p_{i}{ }^{\prime} c(x)\right\} \cdot \tau_{-1}(p)\right. \\
\left.-2 \sum_{i=1}^{n}\left\{p_{i} c(x)\right\} \cdot p_{i} \tau_{-1}(p)\right] f
\end{gathered}
$$

Par récurrence,

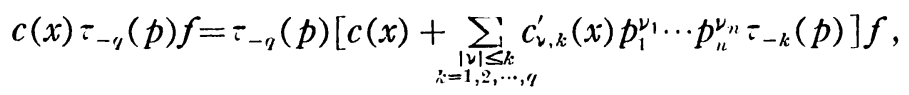

où $c_{v, k}^{\prime}(x)$ sont des combinaisons lineaaires des dérivées de $c(x)$. Comme $f \in\left(\mathscr{D}_{L^{2}}^{\prime}\right)$, on a, pour tout $\varphi(x) \in \mathscr{D}_{L^{2}},\left\langle\tau_{-q} f, \varphi(x)\right\rangle=\langle f$, $\left.\tau_{-1} \varphi(x)\right\rangle$, donc, en considérant deux opérateurs transposés, nous avons $(2 \cdot 1)$.

Démonstration du lemme 2. Pour $s \geq 0$, ce lemme est évident. Pour $s<0$, en remarquant que $D^{-s}$ est le dual de $D^{*}$, et en appliquant à $D^{-\varepsilon}$ ce lemme, on a la propriété voulue.

Définition.

Espace $D_{s}(-\infty<s<+\infty) . \quad F \equiv\left(f_{1}, \cdots, f_{i}, \cdots, f_{m}\right) \in D_{s}$, si $f_{i} \epsilon$ $D^{s-(i-1)}(i=1, \cdots, m)$. On le munit du produit scalaire suivant: $(F, G)=\sum_{i=1}^{m}\left(\tau_{s-(i-1)}(p) f_{i}, g_{i}\right)$.

En introduisant la matrice :

on peut écrire

$$
E_{s}(p)=\begin{array}{ll}
\tau_{s}(p) & \\
& \tau_{s-1}(p) \\
& \dot{\tau}_{s-(m-1)}(p)
\end{array}
$$

$$
(F, G)_{B_{s}}=\left(E_{s}^{1 / 2}(p) F, E_{s}^{1 / 2}(p) G\right)=\left(E_{s}(p) F, G\right) .
$$

Espace $\tilde{D}_{s}(-\infty<s<+\infty) . \quad F \in \tilde{D}_{s}$, si $f_{i} \in D^{s+(i-1)}(i=1, \cdots, m)$. 
On lemunit du produit scalaire svivant:

$$
(F, G) \tilde{D}_{s}=\sum_{i=1}^{m}\left(\tau_{s+(i-1)}(p) \quad f_{i}, g_{i}\right) .
$$

Nous signalons que l'espace $\tilde{D}_{-s}$ est l'espace dual de $D_{s}$, et que la norme de $\tilde{D}_{-s}$ n'est autre que la norme induite comme espace dual par $D_{s}$. En effet, soit $F^{\prime} \in\left(D_{s}^{\prime}\right)$ (espace dual de $D_{s}$ ). Alors, par Riesz, il existe un et un seul élément $F \in D_{s}$ tel que, pour tout $G \in D_{s},\left\langle F^{\prime}, G\right\rangle=(G, F)_{D_{s}}$ c'est-à-dire $=\left(G, E_{s}(p) F\right)$, donc, $F^{\prime}=$ $\overline{E_{s}(p)} F$ ou encore $=E_{s}(\not) \bar{F} \epsilon \tilde{D}_{-s}$. De plus, $\left\|F^{\prime}\right\|_{D_{s}}=\|F\|_{D_{s}}=\|\vec{F}\|_{D_{s}}$ $=\left\|E_{s}^{-1}(p) F^{\prime}\right\|_{D_{s}}=\left\|F^{\prime}\right\|_{\tilde{D}_{-s}}$.

3. Etant donnée une équation kowalewskienne, écrite comme équation d'évolution,

$$
(d / d t)^{\prime \prime} u(t)+\sum_{i=0}^{m-1} \alpha_{i}^{\prime}(x, t, p)(d / d t)^{i} u(t)=v(t),
$$

oú $\alpha_{i}(x, t, p)$ sont des polynòmes en $p$ d'ordre $\leq(m-i)$, dont les coefficients $\alpha_{i, v}(x, t)$ sont des fonctions continument différentiables en $t \grave{a}$ valeurs dans ( $\beta$ ).

Alors, nous dirons d'après Leray ([1], page 128$)$ que $(3 \cdot 1)$ est régulièrement hyperbolique dans $t_{1} \leq t \leq t_{2}$, en posant

$(3 \cdot 2) \quad f(r)=r^{m}+h_{m-1}(x, t, \xi) r^{m-1}+\cdots+h_{1}(x, t, \xi) r+h_{0}(x, t, \xi)=0$

où $h_{i}(x, t, p)$ étant les parties homogènes de degré $(m-i)$ des polynômes $\alpha_{i}(x, t, p)$, si cette équation admet toujours $m$ racines réelles distinctes pour $t \in\left[t_{1}, t_{2}\right], x \in R^{n}$, et pour $\leqslant$ réel $\neq 0$, et de plus, quand $|\xi|=1$, il existe un nombre $\hat{o}$ tel que $\left|\boldsymbol{r}_{i}-\boldsymbol{r}_{j}\right| \geq \boldsymbol{o}>0$ $(i \neq j ; i, j=1, \cdots, m)$, pour tout $t \in\left[t_{1}, t_{2}\right]$, et $x \in R^{\prime \prime}$ fixés, $r_{i}$ étant les $m$ racines de $(3 \cdot 2)$.

$(3 \cdot 1)$ est équivalent à

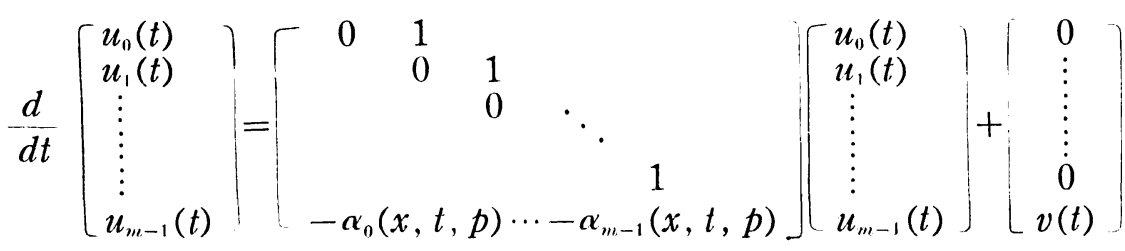

ou

$$
(d / d t) U(t)=A(x, t, p) U(t)+V(t) .
$$

Nous considérerons désormais l'équation sous cette forme, dans l'espace $D_{s}$ ( $s$, entier quelconque). 
Alors, à partir de cette condition d'hyperbolicité, M. Leray a trouvé, pour chaque $l$ entier $>m(m-1) / 2$, une matrice $B_{l}(x, t, p)$ $=\left(b_{i j}(x, t, p)\right)$ telle que 1$) b_{i j}(x, t, p), t_{1} \leq t \leq t_{2}$, sont des polynòmes en $p$ de degré au plus $2 l-(i+j-2)$, avec les coefficients continûment différentiables en $t$ à valeurs dans $\left.(\mathcal{B})_{x}, 2\right) B_{l}(x, t, p)$ est hermitienne positive définie: il existe $\sigma>0$ tel que

$$
B_{l}(x, t, p) \geq \sigma E_{l}(p)
$$

3) il existe $\gamma_{l}>0$ tel que

$$
\begin{aligned}
& -\gamma_{l} E_{l}(p)<B_{l}(x, t, p) A(x, t, p) \\
& +\left[B_{l}(x, t, p) A(x, t, p)\right]^{*}<\gamma_{l} E_{l}(p) .
\end{aligned}
$$

Remarquons que $B_{l}(x, t, p)$ est continûment différentiable en $t$ à valeurs dans $\mathscr{L}\left(D_{s}, \tilde{D}_{s-2 l}\right)$, ou $s$ est un entier quelconque. (Sauf mention expresse, $\mathscr{L}(E, F), E$ et $F$ deux espaces de Banach, est muni de la norme canonique).

Maintenent, nous prenons un $l$, de sorte que $B_{l}(x, t, p)$. Nous voulons en déduire des matrices $B_{l-\cdots q}(x, t, p)(q=1,2, \cdots)$ de la manière suivante:

$$
B_{l-\because y}(x, t, p)=\tau_{-\imath}(p) B_{l}(x, t, p) \tau_{-\imath}(p) .
$$

Alors, $B_{l-\Omega_{l}}(x, t, p)$ est aussi hermitienne, et on a

$$
B_{l-\mathfrak{l} q}(x, t, p) \geq \sigma E_{l-\underline{q} q}(p) .
$$

Nous voulons démontrer qu'il existe $\beta_{\eta}>0$ tel que

$$
-\beta_{\eta} E_{l-\eta \eta}(p) \leq B_{l-\because q} A+\left(B_{l-\because q} A\right)^{*} \leq \beta_{q} E_{l-\because \eta}(p) \text {. }
$$

Démonstration. D'après $(2 \cdot 1)$, posons

$$
\tau_{-\vartheta}(p) A(x, t, p)=\left[A(x, t, p)+A^{\prime}(x, t, p)\right] \tau_{-q}(p),
$$

où $A^{\prime}(x, t, p)$ est une matrice qu'on obtient en remplaçant, dans $A(x, t, p)$, les coefficients $c(x)$ par $\sum_{\substack{|v| \leq k \\ k=1, \cdots, \cdots, n}} p_{1}^{\nu_{1}} \cdots p_{n}^{\nu_{n} \tau_{-k}}(p) c_{\nu, k}^{\prime}(x), c_{\nu, k}^{\prime}(x)$ étant des combinaisons linéaires des dérivées (en $x$ ) de $c(x)$.

Alors, l'opérateur $p_{1}^{\nu_{1}} \cdots p_{n}^{\nu_{n}} \tau_{-k}(p)$ est une application continue de $D^{s}$ dans $D^{i+2 k-\{\nu \mid}$, et comme $2 k-|\nu| \geq 1$, cet opérateur est une application continue de $D^{*}$ dans $D^{*+1}$. En vertu du lemme 2, $A^{\prime}(x, t, p)$ est donc continûment différentiable en $t$ à valeurs dans $\mathscr{L}\left(D_{s}, D_{s}\right)$ (s, entier). Nous avons donc 
Le probleme de Cauchy pour les équations hyperboliques

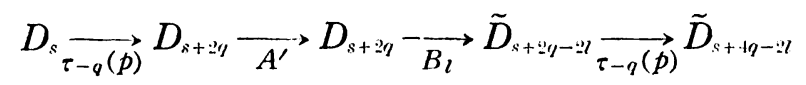

Posons $s=l-2 q$, alors l'opérateur composé est continûment différentiable en $t$ à valeurs dans $\mathscr{L}^{b}\left(D_{l-\because q}, \tilde{D}_{-(l-2 q)}\right)$. Comme $\left(A^{\prime}\right)^{*}$ (l'operateur adjoint de $\left.A^{\prime}\right) \in \mathscr{L}^{\circ}\left(\tilde{D}_{s}, \tilde{D}_{s}\right)$ (s, un entier quelconque), l'opérateur $\left(\overline{-}_{-q} B_{1} A^{\prime} \tau_{-1,}\right) *$ est aussi continûment différentiable en $t$ à

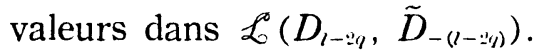

$\tau_{-q}(p)\left[B_{l} A^{\prime}+\left(B_{l} A^{\prime}\right)^{*}\right] \tau_{-q}(p)$, qui est hermitien, est donc majoré et minoré par des constants multiples de $E_{l-2 q}(p)$. Compte tenu de $(3 \cdot 5)$, nous avons $(3 \cdot 8)$.

Si l'on part de $B_{l+1}(x, t, p)$, alors que nous sommes partis de $B_{l}$, on aura des matrices $B_{l-1-2, q}(x, t, p)(q=1,2, \cdots)$ ayant les propriétés $(3 \cdot 7)$ et $(3 \cdot 8)$. Nous avons donc la

Proposition 1. Soit qun entier (quelconque), alors il existe une matrice $B_{q}(x, t, p), t \in\left|t_{1}, t_{2}\right|$, positive hermitienne telle que

i) $B_{q}(x, t, p)$ soit continument différentiable en $t$ à valeurs dans $\mathscr{L}\left(D_{q}, \tilde{D}_{-q}\right)$,

ii) $\sigma^{\prime} E_{\eta}(p) \geq B_{q}(x, t, p) \geq \sigma E_{q}(p)$, pour $t \in\left[t_{1}, t_{2}\right], \sigma, \sigma^{\prime}>0$,

iii) $-r_{q} E_{l}(p)<B_{q} A+\left(B_{q} A\right)^{*}<r_{1} E_{\imath}(p)$, pour $t \in\left[t_{1}, t_{2}\right], r_{l}>0$ étant constante. Ou encore, compte tenu de ii),

iii') $\quad-\gamma_{q}^{\prime} B_{q}(x, t, p)<B_{q} A+\left(B_{q} A\right) *<\gamma_{q}^{\prime} B_{q}(x, t, p)$.

4. Inégalite d'énergie. Comme M. Leray l'a montré, la prop. 1 donne une inégalité pour les solutions de $(3 \cdot 3)$. Soit $q$ un entier. Supposons, dans (3.3), $U(t), U^{\prime}(t), V(t) \in D_{q}\left[t_{1}, t_{2}\right]$, où $D_{q}\left[t_{1}, t_{2}\right]$ est l'espace des fonctions continues en $t, t \in\left[t_{1}, t_{2}\right]$, à valeurs dans $D_{q}$, muni de la topologie uniforme en $t$. Alors, le raisonnement de [1], p. 131, et la prop. 1 nous donnent l'inégalité d'énergie suivante :

$$
\begin{aligned}
& \sqrt{\left(B_{q} U, U\right)_{t}} \leq \int_{t_{0}}^{t} \sqrt{\left(B_{q} V, V\right)_{s}} \exp r(t-s) d s \\
& +\sqrt{\left(B_{l} U, U\right)_{t_{0}}} \exp r\left|t-t_{0}\right|, \quad r>0
\end{aligned}
$$

pour $t_{1} \leq t \leq t_{2}, t_{1}$ étant une valeur quelconque dans $\left[t_{1}, t_{2}\right]$.

5. Problème de Cauchy. Considérons l'équation

$$
(d / d t) U(t)=A(x, t, p) U(t)+V(t),
$$

dans l'espace $D_{q}$ ( $q$, un entier quelconque). Nous supposons que la donnée initiale $U_{v} \in D_{q^{+1}}$, et que $V(t) \in D_{q+1}\left[t_{1}, t_{2}\right]$. Alors, il existe 
une suite $V_{j}(t) \in \mathcal{D}_{L^{2}}\left[t_{1}, t_{2}\right]$, tendant vers $V(t)$ pour la topologie de $D_{q+1}\left[t_{1}, t_{\mathrm{z}}\right]$. Voir [1], p. 135. Nous donnons la démonstration.

A) Soit $f \in D^{*}(-\infty<s<+\infty)$, alors $f_{\delta}(x)=f^{*}(2 \pi \grave{o})^{-n / 2} \exp$ $\left(-x^{2} / 2 i\right), i>0$, converge vers $f$ lorsque $i \rightarrow 0$, pour la topologie de $D^{s}$. Démonstration. La transformée de Fourier de $f_{\delta}(x)$ devient $\varphi_{0}(\xi) \equiv \varphi(\xi) \exp \left(-2 \pi^{2}|\xi|^{2} \grave{o}\right)$, où $\varphi(\xi)=\not{f}(f)$. Il suffit de démontrer que

$$
\left(1+4 \pi^{2}|\xi|^{2}\right)^{\xi / 2} \varphi_{\delta}(\xi) \underset{\delta \rightarrow 0}{\longrightarrow}\left(1+\left.\left.4 \pi^{2}\right|^{\xi}\right|^{2}\right)^{s / 2} \varphi(\xi) \epsilon\left(L^{2}\right)_{\xi},
$$

pour la topologie de $L^{2}$. Or, ceci résulte du fait que, $\psi^{\prime}(\xi)$ étant dans $L^{2}$,

$$
\left.\int \cdots \int\left|1-\exp \left(-2 \pi^{2}|\hat{\xi}|^{2} \boldsymbol{o}\right)\right|^{2} \cdot \mid \zeta^{(\xi)}\right)\left.\right|^{2} d \xi \underset{\delta \rightarrow 0}{\longrightarrow} 0,
$$

ce qui serait évident.

B) Le raisonnement dans A) montre que, soit $f(t) \in D^{*}[a, b]$, $(-\infty<s<\infty)$, alors $f_{j}(t) \in \mathscr{D}_{t^{2}}[a, b], f_{j}(t)_{(x)}^{*}\left(2 \pi \hat{o}_{j}\right)^{-n / 2} \exp \left(-x^{2} / 2 \dot{o}_{j}\right)$, $\partial_{j} \rightarrow 0$ converge vers $f(t)$ pour la topologie de $D^{r}[a, b]$. En effet,

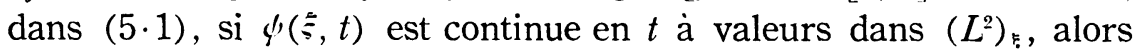
la convergence aura lieu uniformément en $t$.

Considérons la suite $U_{j}(t),(j=1,2, \cdots)$, où $U_{j}(t)$ est la solution de l'équation

$(d / d t) U_{j}(t)=A(x, t, p) U_{j}(t)+V_{j}(t)$, avec la condition initiale $U_{j}\left(t_{0}\right)=U_{n, j}$, où $V_{j}(t)$ et $U_{n, j}$ sont des suites convergeant vers $V(t)$ et $U_{0}$ pour la topologie de $D_{\eta+1}\left[t_{1}, t_{2}\right]$ et de $D_{q+1}$ respectivement. Or, $U_{j}(t)$ est une suite de Cauchy pour la topologie de $D_{q+1}\left[t_{1}, t_{2}\right]$. En effet, $U_{i}(t)-U_{j}(t)$ est la solution avec la condition initiale $U_{1, i}$ $-U_{0, j}$ et avec le second membre $F(t)=V_{i}(t)-V_{j}(t)$. Donc, l'inégalite $(4 \cdot 1)$ montre que $U_{f}(t) \rightarrow U^{(\infty)}(t)$ pour la topologie de $D_{\eta^{+1}}$ $\left[t_{1}, t_{2}\right]$.

Or,

$$
U_{j}(t)=U_{i, j}+\int_{i_{10}}^{t}\left[A(x, t, p) U_{j}(t)+V_{j}(t)\right] d t
$$

montre que, comme la convergence $A(x, t, p) U_{j}(t) \rightarrow A(x, t, p) U^{(\infty)}(t)$ a lieu pour la topoiogie de $D_{q}\left[t_{1}, t_{2}\right]$, (car, $A(x, t, p)$ est continue en $t$ à valeurs dans $\left.\mathscr{L}^{\mathbb{b}}\left(D_{q_{+1}}, D_{q}\right)\right), U^{(\infty)}(t)$ est donc une solution de $(3 \cdot 3)$, continûment différentiable pour la topologie de $D_{q}$, avec la condition initiale: $U\left(t_{0}\right)=U_{0}$. Comme l'unicité de la solution résulte de $(4 \cdot 1)$, nous avons donc la 
Proposition 2. Etant donnés dans (3.3) la condition initiale $U_{v} \in D_{q+1}$, pour $t=t_{0}, t_{1} \leq t_{0} \leq t_{2}$, et le second membre $V(t) \in D_{\eta+1}\left[t_{1}\right.$, $t_{2}$ ], oì $q$ est un entier (positif, o, négatif), il existe alors une et une seule solution $U(t)$ telle que

i) elle soit continument différentiable pour la topologie de $D_{q}$,

ii) $\left.U(t) \in D_{\imath+1} \mid t_{1}, t_{2}\right], U\left(t_{0}\right)=U_{0}, t_{1} \leq t_{0} \leq t_{2}$.

Revenons à l'équation $(3 \cdot 1)$, et considérons les solutions dans l'espace $D^{\prime}$ ( $q$, entier quelconque), alors la prop. 2 se traduit de la manière suivante.

PROPOSITION 3. Etant donnés la condition initiale pour $t=t_{0}$, $t_{0} \in\left[t_{1}, t_{2}\right]$, et le second membre $v(t):\left(u_{0}, u_{1}, \cdots, u_{m-1}\right) \in\left(D^{q+m}\right.$, $\left.D^{q+(m-1)}, \cdots, D^{q+1}\right)$, et $v(t) \in D^{\eta+1}\left[t_{1}, t_{2}\right]$, alors il existe une et une seule solution $u(t), t_{1} \leq t \leq t_{2}$, telle que de $D^{\prime}$,

i) elle est $m$ fois continûment différentiable pour la topologie

ii) $\quad(d / d t)^{i} u(t) \in D^{q+(m-i)}\left[t_{1}, t_{2}\right]$, $(d / d t)^{i} u\left(t_{0}\right)=u_{i}, \quad(i=0,1, \cdots, m-1)$.

6. Extension à des espaces des distributions tempérées. Nous allons montrer qu'une extension de nos résultats (les prop. 2, 3) à des espaces des distributions tempérées est immédiate.

Lemma 3. Soit $k$ un entier positif. Alors

$$
\frac{1}{\left(1+r^{2}\right)^{k}} A(x, t, p)=\left[A(x, t, p)+R_{1}(x, t, p)\right] \frac{1}{\left(1+r^{2}\right)^{k}}
$$

oì $R_{1}(x, t, p)=\left(r_{i j}(x, t, p)\right), r_{i j}(x, t, p)=0$ pour $i \neq m$, $r_{m j}(x, t, p)=u n$ polynôme en $p$ de degré $\leq(m-j)$, avec des coefficients continument différentiables en $t$ à valeurs dans ( $\mathcal{B})_{x}$.

Démonstration. Pour $|\lambda|>0$, on aura la formule suivante qui est facile à vérifier.

$$
\begin{gathered}
\frac{1}{\left(1+r^{2}\right)^{k}} p_{1}^{\lambda_{1}} \cdots p_{n}^{\lambda_{n}} f=p_{1}^{\lambda_{1}} \cdots p_{n}^{\lambda_{n}} \frac{1}{\left(1+r^{2}\right)^{k}} f \\
\quad+\sum_{|\mu,| \geq 1} c_{\lambda, \mu}(x) p_{1}^{\lambda_{1}-\mu_{1} \cdots} p_{u}^{\lambda_{n}-\mu_{n}} \frac{1}{\left(1+r^{2}\right)^{k}} f,
\end{gathered}
$$

où $c_{\lambda, \mu}(x) \in(\beta)$.

Considérons l'équation

c. q.f.d.

$$
(d / d t) U(t)=A(x, t, p) U(t)+V(t) .
$$


Appliquons $1 /\left(1+r^{2}\right)^{k}(k>0$ entier) à gauche, alors, en vertu du lemme précédent,

$$
\begin{aligned}
\frac{d}{d t} \frac{1}{\left(1+r^{2}\right)^{k}} U(t)= & {\left[A(\dot{x}, t, p)+R_{1}(x, t, p)\right] \frac{1}{\left(1+r^{2}\right)^{k}} U(t) } \\
& +\frac{1}{\left(1+r^{2}\right)^{k}} V(t) .
\end{aligned}
$$

Le lemme 3 montre que $A+R_{1}$ est également régulièrement hyperbolique, car l'oprateur $R_{1}$ n'influence pas la condition d'hyperbolicité. On peut donc appliquer à cet opérateur les prop. 2, 3 . Nous nous bornons à énoncer la proposition suivante, $q$ étant un entier (positif, négatif ou zéro).

Proposition 4. Etant donnés $U_{\mathrm{v}}$ et $V(t)$ tels que $\frac{1}{\left(1+r^{2}\right)^{k}} U_{0}$ $\epsilon D_{q^{+1}}, \frac{1}{\left(1+r^{2}\right)^{k}} V(t) \in D_{\eta_{+1}}\left[t_{1}, t_{2}\right]$, alors il existe une et une seule solution $U(t), t \in\left[t_{1}, t_{2}\right]$, de l'équation (3.3) telle que

i) $\frac{1}{\left(1+r^{2}\right)^{k}} U(t) \in D_{q+1}\left[t_{1}, t_{2}\right], U\left(t_{0}\right)=U_{0} ; t_{1} \leq t_{0} \leq t_{2}$,

ii) $\frac{1}{\left(1+r^{2}\right)^{k}} U(t)$ est continument différentiable en $t$ pour la topologie de $D_{q}$.

\section{BIBLIOGRAPHIE}

[1] J. Leray, Hyperbolic differential equations (cours de Princeton) 1954.

[2] L. Schwartz, Les équations d'évolution liées au produit de compositions, Ann. Inst. Fourie. 1950.

[3] L. Schwarts, Théorie des distributions, Paris, Hermann, 1950-51. 